\title{
Analysis of Transient Pulse Electroosmotic Flow of Maxwell Fluid through a Circular Micro-Channel Using Laplace Transform Method
}

\author{
Dongsheng Li, Kun Li* \\ College of Science, Inner Mongolia University of Technology, Hohhot, China \\ Email: *jadewh@163.com
}

How to cite this paper: Li, D.S. and Li, K. (2021) Analysis of Transient Pulse Electroosmotic Flow of Maxwell Fluid through a Circular Micro-Channel Using Laplace Transform Method. Open Journal of Fluid Dynamics, 11, 67-80.

https://doi.org/10.4236/ojfd.2021.112005

Received: March 6, 2021

Accepted: May 7, 2021

Published: May 10, 2021

Copyright $\odot 2021$ by author(s) and Scientific Research Publishing Inc. This work is licensed under the Creative Commons Attribution International License (CC BY 4.0).

http://creativecommons.org/licenses/by/4.0/ (c) (i) Open Access

\begin{abstract}
A semi-analytical solution is presented using method of Laplace transform for the transient pulse electroosmotic flow (EOF) of Maxwell fluid in a circular micro-channel. The driving mode of pulse EOF here is considered as an ideal rectangle pulse. The solution involves solving the linearized Poisson-Boltzmann (P-B) equation, together with the Cauchy momentum equation and the general Maxwell constitutive equation. The results show that the profiles of pulse EOF velocity vary rapidly and gradually stabilize as the increase of time $\bar{t}$ within a half period. The velocity profiles at the center of the micro-channel increase significantly with relaxation time $\bar{\lambda}_{1}$, especially for the smaller pulse width $a$. However, as the pulse width $a$ increases, this change will be less obvious. At the same time, the different change frequency of velocity profiles will slow down, which means a long cycle time. Additionally, the time needed to attain the steady status becomes longer with the increase of relaxation time $\bar{\lambda}_{1}$ and pulse width $a$.
\end{abstract}

\section{Keywords}

Pulse Electroosmotic Flow, Laplace Transform, Maxwell Fluid, Relaxation

Time, Pulse Width

\section{Introduction}

In recent years, microfluidic devices have been vigorously developed and applied in micro-electronic mechanical systems and microbiological sensors, and the electroosmotic flow (EOF) formed in these devices has become more and more 
attractive [1] [2]. Different from the flow in the conventional macro-sized channel, the existence of the electric double layer (EDL) must be considered when the flow is analyzed in the micro-channel [3]. Generally, when most substances come into contact with polar solutions, they tend to generate negative charges on the surface. The distribution of ions near the wall in the solution will be affected by this phenomenon. The opposite ions with the opposite polarity to the wall will be attracted to the wall, while the same ions will be repelled away from the wall. In this way, an electric double layer (EDL) will be formed [4]. Further, when an external electric field is applied to both ends of the channel, the ions in the EDL will move under the force of the electric field. This is due to the viscosity of the fluid itself, the moving free ions will drive the movement of nearby fluid clusters, and eventually form an electroosmotic flow (EOF).

Among previous research, various theoretical and experimental studies on steady EOF of Newtonian fluids in micro-channels under different geometric regions and physical conditions have been carried out [5]-[10]. Recently, time-dependent EOF as an alternative mechanism of microfluidic transport has attracted more and more attention [11]-[17].

All of the above-mentioned studies are related to Newtonian fluids. However, most of solutions of industry and biopharmaceutical are fluid that has the structural characteristic of non-Newtonian fluids, for example biological fluid and other solutions of long-chain molecules, whose structural characteristics include strain force, normal shear stress, hysteresis effect, variable viscosity, memory effect and so on [18]. Therefore, these fluids cannot be regarded as Newtonian fluids. From many literatures, we know that the research on non-Newtonian fluids at home and abroad mainly focuses on the rheological properties of non-Newtonian fluids, non-Newtonian fluids gas-liquid two-phase flow, non-Newtonian fluids mass and heat transfer, and numerical simulation methods of non-Newtonian fluids. Furthermore, the theoretical research of EOF of non-Newtonian fluids is mainly limited to simple fluid models because of the inherent analytical difficulties introduced by more complex constitutive equations [19]. So far, some work has been done on the simple non-Newtonian fluids models, such as Oldroyd-B fluid model [20] [21] [22], Power-law fluid model [23] [24] [25], Maxwell fluid model [26]-[32] and Jeffrey fluid model [33] [34] [35].

Although we have learned some basic characteristics of EOF of non-Newtonian fluids in the above research, its rich characteristics still need to be studied. Recent study have shown that Maxwell fluid model simulation of blood in narrow conical vessels has achieved an ideal effect, and it is completely possible to analyze the blood-based microfluidics and other microbial fluid transmission systems by means of electric mechanism [26]. Meanwhile, the literature [36] found that low-frequency pulses can stimulate nerve cell tissues, relieve pain and promote local blood circulation, which has been confirmed in clinical medical research. Additionally, the circular channel is very common in practical applications [34]. So we select Maxwell fluid model as a constitutive relation to describe 
pulse EOF in the circular micro-channel in present work.

Besides, Liu et al. [37] studied the alternating current (AC) EOF of generalized Maxwell fluid through a circular micro-channel and an analytical solution of EOF velocity distribution is derived. Taking into account the limitations of alternating current (such as continuous and time-varying), as well as the wide application of pulse current in engineering in recent years [38] [39] [40], thus another important purpose of this paper is also extending the AC EOF to pulse current (PC) EOF of the generalized Maxwell fluid model in the circular micro-channel. With the help of Laplace transform method, a semi-analytical solution for pulse EOF velocity is obtained by solving the general Cauchy momentum equation. Moreover, we analyzed the influence of several parameters such as the relaxation time, the pulse width and the electrokinetic width on the pulse EOF of Maxwell fluid. The rest of this paper is organized as follows: The physical description of the problem and the analytical solution to the equations governing the pulse EOF of the Maxwell fluid model in a circular micro-channel are given in Section 2. Section 3 discusses the numerical results of the study and the conclusions are presented in Section 4.

\section{Mathematical Formulation}

\subsection{Cauchy Momentum Equation and Constitutive Relation}

The transient pulse EOF of an incompressible Maxwell fluid through a circular micro-channel with radius $R$, the length of the channel is $L$, assumed to be much larger than the diameter i.e., $L \gg 2 R$ is sketched in Figure 1(a). The pulse EOF is pumped through an ideal rectangle pulse electric field of strength $E_{0}$ with pulse amplitude of 1 , pulse repetition period of $2 a$ and pulse width of $a$ in Figure 1(b). The ideal rectangle pulse can be expressed as the following form [41]

$$
f(t)= \begin{cases}1, & t \in[0, a), \\ -1, & t \in(a, 2 a] .\end{cases}
$$

Due to the symmetry of the geometry, we only study the semi-section of the micro-channel. Considering the pure pulse EOF and ignoring the pressure gradient, the one-dimensional Cauchy momentum equation can be given as

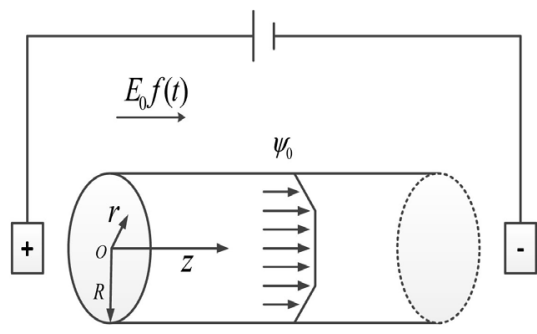

(a)

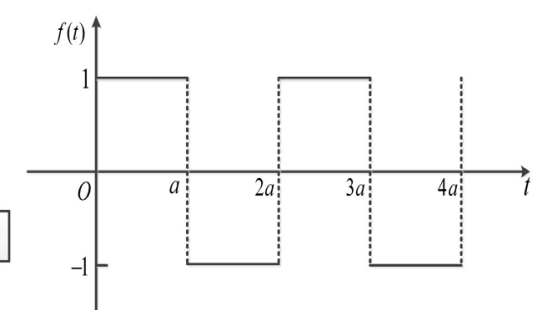

(b)

Figure 1. Schematic of the transient pulse EOF of Maxwell fluid in a circular micro-channel. (a) Circular micro-channel; (b) Time periodic pulse wave (ideal rectangle pulse wave). 


$$
\rho \frac{\partial u(r, t)}{\partial t}=-\frac{1}{r} \frac{\partial}{\partial r}\left(r \tau_{r z}\right)+\rho_{e}(r) E_{0} f(t)
$$

where $u(r, t)$ is the velocity along $z$ axial direction, $\rho$ is the fluid density, $t$ is the time, $\tau_{r z}$ is the stress tensor and $\rho_{e}(r)$ is the volume charge density, $E_{0} f(t)$ is the ideal rectangle pulse electric field of strength $E_{0}$.

The boundary condition of Equation (2) is supposed no slip and can be written as

$$
\left.u(r, t)\right|_{r=R}=0,\left.\frac{\partial u(r, t)}{\partial r}\right|_{r=0}=0
$$

For the Maxwell fluid, the constitutive equation satisfies [42]

$$
\tau_{r z}+\lambda_{1} \frac{\partial}{\partial t} \tau_{r z}=-\eta_{0} \frac{\partial u(r, t)}{\partial r}
$$

where $\lambda_{1}$ and $\eta_{0}$ are the relaxation time and the zero shear rate viscosity, respectively.

\subsection{Electric Potential Field Solution}

The chemical interaction of electrolyte liquid and solid wall generates an electric double layer (EDL), a very thin charged liquid layer at the solid-liquid interface. A cylindrical coordinate system $(r, \theta, z)$ is introduced. In this theoretical model, it is assumed that the channel wall is uniformly charged, so that the electrical potential in the EDL only varies in this $r$ direction and does not depend on $\theta$ [43]. For a symmetric binary electrolyte solution, we assume that the electrical potential $\psi$ of the EDL is stable, and its distribution and the local volumetric net charge density $\rho_{e}(r)$ are given by the Poisson-Boltzmann (P-B) equation

$$
\begin{gathered}
\frac{1}{r} \frac{\mathrm{d}}{\mathrm{d} r}\left(r \frac{\mathrm{d} \psi(r)}{\mathrm{d} r}\right)=-\frac{\rho_{e}(r)}{\varepsilon} \\
\rho_{e}(r)=-2 n_{0} z_{v} e_{0} \sinh \left[\frac{z_{v} e_{0} \psi(r)}{k_{b} T}\right]
\end{gathered}
$$

where $\varepsilon$ is the dielectric constant of the electrolyte liquid, $\psi(r)$ is the electrical potential of the EDL, $n_{0}$ is the ion density of the bulk liquid, $z_{v}$ is the valence, $e_{0}$ is the electron charge, $k_{b}$ is the Boltzmann constant, $T$ is the absolute temperature and sinh is a sine function.

Combining Equations (5) and (6) gives

$$
\frac{1}{r} \frac{\mathrm{d}}{\mathrm{d} r}\left(r \frac{\mathrm{d} \psi(r)}{\mathrm{d} r}\right)=\frac{2 n_{0} z_{v} e_{0}}{\varepsilon} \sinh \left[\frac{z_{v} e_{0} \psi(r)}{k_{b} T}\right]
$$

which is subject to the following boundary conditions

$$
\left.\psi(r)\right|_{r=R}=\psi_{0},\left.\frac{\mathrm{d} \psi(r)}{\mathrm{d} r}\right|_{r=0}=0
$$

where $\psi_{0}$ is wall zeta potential, $r$ is radial coordinate and $R$ is radius of the circular micro-channel. 
Provided that the electrical potential is small enough, the Debye-Hückel linearization approximation can be applied, which means physically that the electrical potential is small compared to the thermal energy of the charged species [33]. Then Equation (7) can be simplified as

$$
\frac{1}{r} \frac{\mathrm{d}}{\mathrm{d} r}\left(r \frac{\mathrm{d} \psi(r)}{\mathrm{d} r}\right)=\kappa^{2} \psi(r), \text { where } \kappa=\left(\frac{2 n_{0} z_{v}^{2} e_{0}^{2}}{\varepsilon k_{b} T}\right)^{1 / 2}
$$

where $\kappa$ is the Debye-Hückel parameter and $1 / \kappa$ usually denotes the thickness of the EDL in physical.

The net charge density can be obtained by solving Equation (9) with boundary condition of Equation (8)

$$
\rho_{e}(r)=-\varepsilon \kappa^{2} \psi_{0} \frac{I_{0}(\kappa r)}{I_{0}(\kappa R)}
$$

where $I_{0}$ is first kind modified Bessel function of order zero.

\subsection{The Analytical Solutions of the Cauchy Momentum Equation}

In order to obtain the solution of velocity field, some dimensionless parameters are given as

$$
\begin{aligned}
& \bar{r}=\frac{r}{R}, K=\kappa R,\left(\bar{t}, \overline{\lambda_{1}}\right)=\frac{\left(t, \lambda_{1}\right)}{\rho R^{2} / \eta_{0}} \\
& \bar{u}(\bar{r}, \bar{t})=\frac{u(r, t)}{U_{e o}}, \overline{\tau_{\overline{r z}}}=\frac{\tau_{r z}}{\eta_{0} U_{e o} / R}, U_{e o}=-\frac{\varepsilon \psi_{0} E_{0}}{\eta_{0}}
\end{aligned}
$$

where $U_{\text {eo }}$ denotes dimensionless steady Helmholtz-Smoluchowshi EOF velocity of Newtonian fluids, $K$ is the ratio of the characteristic width of the micro-channel to Debye length.

Using Equation (11), the Equations of (2) and (4) and the corresponding boundary condition (3) can be written as

$$
\begin{gathered}
\frac{\partial \bar{u}(\bar{r}, \bar{t})}{\partial \bar{t}}=-\frac{1}{\bar{r}} \frac{\partial}{\partial \bar{r}}\left(\bar{r} \overline{\tau_{\overline{r z}}}\right)+K^{2} f(\bar{t}) \frac{I_{0}(K \bar{r})}{I_{0}(K)} \\
\overline{\tau_{\overline{r z}}}+\overline{\lambda_{1}} \frac{\partial}{\partial \bar{t}} \overline{\tau_{\overline{r z}}}=-\frac{\partial \bar{u}(\bar{r}, \bar{t})}{\partial \bar{r}} \\
\left.\bar{u}(\bar{r}, \bar{t})\right|_{\bar{r}=1}=0,\left.\frac{\partial \bar{u}(\bar{r}, \bar{t})}{\partial \bar{r}}\right|_{\bar{r}=0}=0
\end{gathered}
$$

Eliminating $\overline{\tau_{\overline{r z}}}$ from Equation (12) and Equation (13) yields

$$
\begin{aligned}
& \frac{\partial \bar{u}(\bar{r}, \bar{t})}{\partial \bar{t}}+\bar{\lambda}_{1} \frac{\partial^{2} \bar{u}(\bar{r}, \bar{t})}{\partial \bar{t}^{2}} \\
& =\frac{1}{\bar{r}} \frac{\partial}{\partial \bar{r}}\left(\bar{r} \frac{\partial \bar{u}(\bar{r}, \bar{t})}{\partial \bar{r}}\right)+\left(1+\bar{\lambda}_{1} \frac{\partial}{\partial \bar{t}}\right) K^{2} f(\bar{t}) \frac{I_{0}(K \bar{r})}{I_{0}(K)}
\end{aligned}
$$

Let us employ the method of Laplace transform defined by

$$
U(\bar{r}, s)=L[\bar{u}(\bar{r}, \bar{t})]=\int_{0}^{\infty} \bar{u}(\bar{r}, \bar{t}) \mathrm{e}^{-s \bar{t}} \mathrm{~d} \bar{t}
$$


Obviously $\frac{\partial}{\partial \bar{t}} f(\bar{t})=0$, and the Laplace transform of $f(\bar{t})$ is given by the Appendix. If initial condition satisfies $\bar{u}(\bar{r}, 0)=0$, the Laplace transforms of Equation (15) and boundary condition (14) can be given as

$$
\begin{gathered}
\bar{\lambda}_{1} s^{2} U(\bar{r}, s)+s U(\bar{r}, s)=\frac{\partial^{2} U(\bar{r}, s)}{\partial \bar{r}^{2}}+\frac{1}{\bar{r}} \frac{\partial U(\bar{r}, s)}{\partial \bar{r}}+\frac{K^{2} \tanh \left(\frac{a s}{2}\right)}{s} \frac{I_{0}(K \bar{r})}{I_{0}(K)} \\
\left.U(\bar{r}, s)\right|_{\bar{r}=1}=0,\left.\frac{\partial U(\bar{r}, s)}{\partial \bar{r}}\right|_{\bar{r}=0}=0
\end{gathered}
$$

here tanh is a hyperbolic tangent function.

Equation (17) can be simplified as

$$
\frac{\partial^{2} U(\bar{r}, s)}{\partial \bar{r}^{2}}+\frac{1}{\bar{r}} \frac{\partial U(\bar{r}, s)}{\partial \bar{r}}-\beta^{2} U(\bar{r}, s)=-\frac{K^{2} \tanh \left(\frac{a s}{2}\right)}{s} \frac{I_{0}(K \bar{r})}{I_{0}(K)}
$$

where $\beta=\sqrt{\bar{\lambda}_{1} s^{2}+s}$.

Equation (19) is a linear and inhomogeneous ordinary differential equation, and its solution can be written as the sum of a general solution $U_{h}(\bar{r}, s)$ corresponding to homogeneous equation and a special solution $U_{s}(\bar{r}, s)$.

$$
U(\bar{r}, s)=U_{h}(\bar{r}, s)+U_{s}(\bar{r}, s)
$$

Due to the finite of $U(\bar{r}, s)$ at $\bar{r}=0$, the homogeneous solution of Equation (19) is written as

$$
U_{h}(\bar{r}, \bar{s})=A I_{0}(\beta \bar{r})
$$

here $A$ is constant, which can be determined from boundary conditions of Equation (18).

Observing the formation of the right hand side of Equation (19), the special solution can be expressed as

$$
U_{s}(\bar{r}, s)=C I_{0}(K \bar{r})
$$

Substituting Equation (22) into Equation (19) yields

$$
C\left[\frac{\mathrm{d}^{2} I_{0}(K \bar{r})}{\mathrm{d} \bar{r}^{2}}+\frac{1}{\bar{r}} \frac{\mathrm{d} I_{0}(K \bar{r})}{\mathrm{d} \bar{r}}-\beta^{2} I_{0}(K \bar{r})\right]=-\frac{K^{2} \tanh \left(\frac{a s}{2}\right)}{s} \frac{I_{0}(K r)}{I_{0}(K)}
$$

From Equation (9) and Equation (11), we have

$$
\frac{\mathrm{d}^{2} I_{0}(K \bar{r})}{\mathrm{d} \bar{r}^{2}}+\frac{1}{\bar{r}} \frac{\mathrm{d} I_{0}(K \bar{r})}{\mathrm{d} \bar{r}}=K^{2} I_{0}(K \bar{r})
$$

Inserting Equation (24) into Equation (23) and equalizing the coefficients in front of the modified Bessel functions $I_{0}(K \bar{r})$ at the two sides of the equation yields

$$
C=-\frac{K^{2} \tanh \left(\frac{a s}{2}\right)}{s\left(K^{2}-\beta^{2}\right) I_{0}(K)}
$$


Therefore, the solution of velocity $U(\bar{r}, \bar{s})$ can be given as

$$
U(\bar{r}, s)=A I_{0}(\beta \bar{r})-\frac{K^{2} \tanh \left(\frac{a s}{2}\right)}{s\left(K^{2}-\beta^{2}\right) I_{0}(K)} I_{0}(K \bar{r})
$$

The coefficient $A$ with boundary condition of Equation (18) can be determined as

$$
A=\frac{K^{2} \tanh \left(\frac{a s}{2}\right)}{s\left(K^{2}-\beta^{2}\right) I_{0}(\beta)}
$$

Substituting Equation (27) into Equation (26), we can get

$$
U(\bar{r}, s)=\frac{K^{2} \tanh \left(\frac{a s}{2}\right)}{s\left(K^{2}-\beta^{2}\right)}\left(\frac{I_{0}(\beta \bar{r})}{I_{0}(\beta)}-\frac{I_{0}(K \bar{r})}{I_{0}(K)}\right)
$$

The inverse Laplace transform is defined by

$$
\bar{u}(\bar{r}, \bar{t})=L^{-1}[U(\bar{r}, s)]=\frac{1}{2 \pi i} \int_{\Gamma} U(\bar{r}, s) \mathrm{e}^{s \bar{t}} \mathrm{~d} s
$$

where $\Gamma$ is a vertical line to the right of all singularities of $U(\bar{r}, s)$ in the complex $s$ plane. Because of the complexity of the express of $U(\bar{r}, s)$, the numerical computation must be performed by numerical inverse Laplace transform [44]. The method of Laplace inverse transform in the above-mentioned literature needs to be supplemented by the fact that the Laplace inverse transform is integrated using the trapezoidal rule, and the Laplace numerical inverse transform is based on the Fourier series that accelerates the convergence.

\section{Results and Discussion}

In the second section, we have obtained the semi-analytical solution of the transient pulse EOF velocity of Maxwell fluid through a circular micro-channel, which mainly relies on relevant dimensionless parameters, such as the relaxation time $\bar{\lambda}_{1}$, the pulse width $a$ and the electrokinetic width $K$. In this section, we will discuss their influence on the normalized pulse EOF velocity in detail.

Figure 2 shows the variations of normalized pulse EOF velocity with radius in one cycle under different relaxation time $\bar{\lambda}_{1}(0.01,0.05,0.1$ and 0.2$)$. It can be seen from Figure 2 that for fixed electrokinetic width $K$ and pulse width $a$, the amplitude of the normalized pulse EOF velocity profiles varies significantly with the increase of relaxation time $\bar{\lambda}_{1}$, and the velocity profiles varies as the time $\bar{t}$ increases. For fixed relaxation time $\bar{\lambda}_{1}$, the fluid velocity will gradually stabilize with the increase of time $\bar{t}$ within a half period. The larger the relaxation time $\bar{\lambda}_{1}$, the larger the amplitude of the velocity profiles, and the longer the time it takes for the fluid to reach a steady status. The main reason of this fact is that for larger relaxation time, the distorted polymer molecules will have no time to change their structure during the time scale of the fluid flow, and the behavior of 


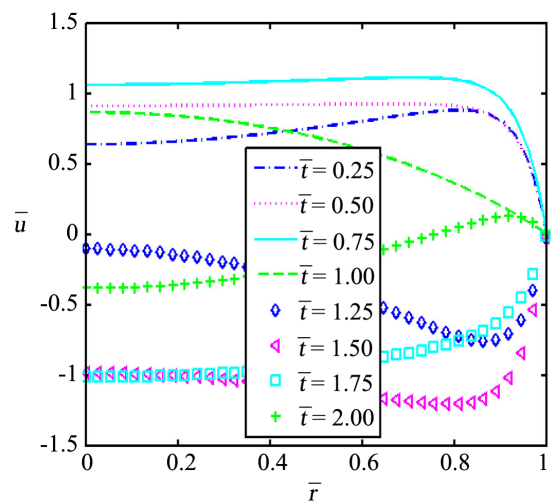

(a)

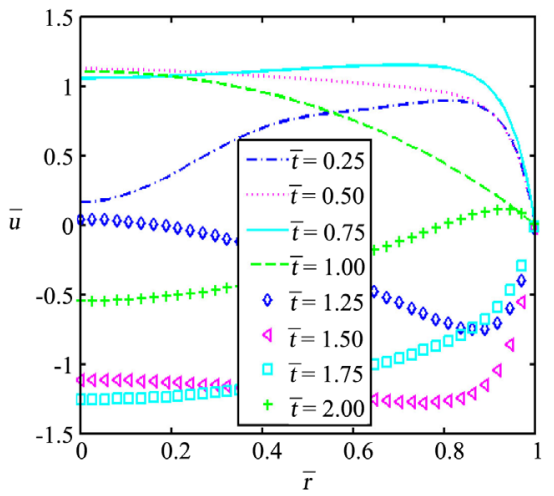

(c)

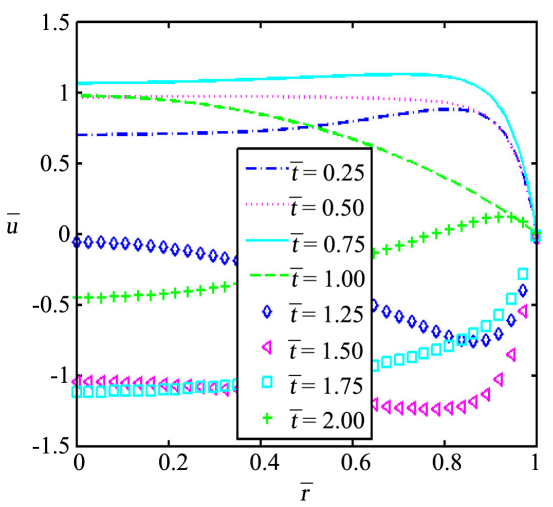

(b)

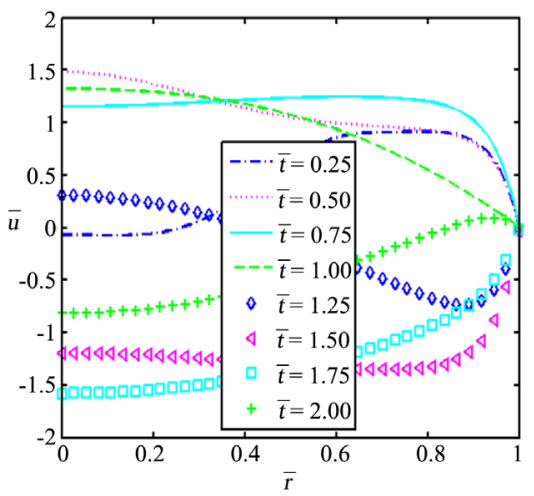

(d)

Figure 2. Variation of normalized pulse EOF velocity at different time with radius for different relaxation time $\bar{\lambda}_{1}$ when $K=20$ and $a=1$. (a) $\bar{\lambda}_{1}=0.01$; (b) $\bar{\lambda}_{1}=0.05$; (c) $\bar{\lambda}_{1}=0.1 ;(\mathrm{d}) \quad \bar{\lambda}_{1}=0.2$.

the fluid gradually tends to become a Hookean elastic solid. Thus, the flow takes longer time to attain the steady status [45].

Figure 3 depicts the variations of normalized pulse EOF velocity with time for different relaxation time $\bar{\lambda}_{1}(0.1,0.3,0.5$ and 0.7$)$. As expected, we can find that for fixed pulse width $a$, the amplitude of the normalized pulse EOF velocity profiles at the center of the micro-channel (that is, $\bar{r}=0.5$ ) increases significantly with the increase of the relaxation time $\bar{\lambda}_{1}$, especially for the smaller pulse width $a$ (see Figures 3(a)-(c)). However, for larger pulse width $a$, this change will be less obvious. This implies that increasing the pulse width will weaken the effect of relaxation time on velocity. The reason may be a larger pulse width $a$, which means that the longer the pulse force lasts, the stronger its stability. For fixed relaxation time $\bar{\lambda}_{1}$, the normalized pulse EOF velocity profiles of frequency are different due to the different pulse width $a$. With the pulse width $a$ increases, the different change frequency of velocity profiles will slow down, which means a long cycle time [41], and the time required for the velocity profiles to reach a steady state becomes longer.

The variations of normalized pulse EOF velocity with radius for different relaxation time $\bar{\lambda}_{1}(0.01,0.1,0.3$ and 0.7$)$ are presented in Figure 4. For a smaller 


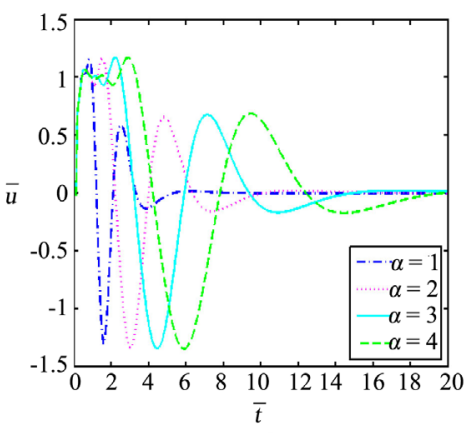

(a)

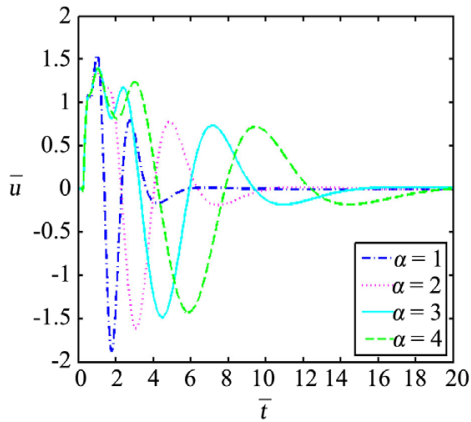

(c)

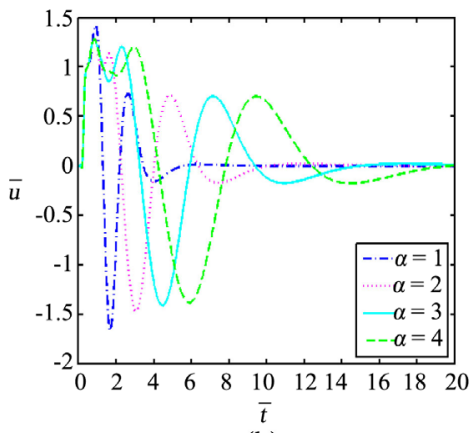

(b)

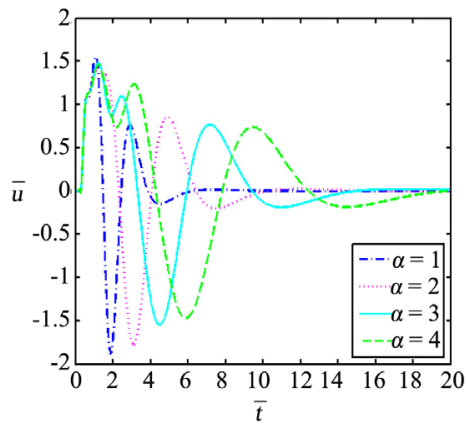

(d)

Figure 3. Variation of normalized pulse EOF velocity at different pulse width with time for different relaxation time $\bar{\lambda}_{1}$ when $K=20$ and $\bar{r}=0.5$. (a) $\bar{\lambda}_{1}=0.1$; (b) $\bar{\lambda}_{1}=0.3$; (c) $\bar{\lambda}_{1}=0.5 ;$ (d) $\quad \bar{\lambda}_{1}=0.7$.

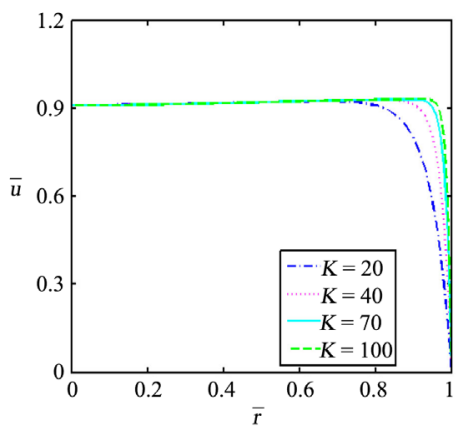

(a)

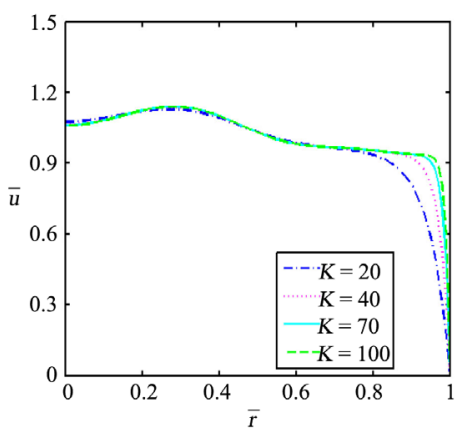

(c)

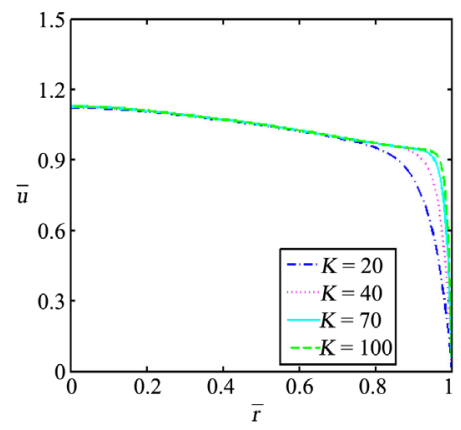

(b)

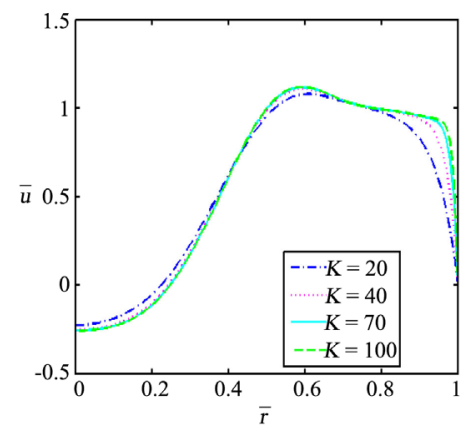

(d)

Figure 4. Variation of normalized pulse EOF velocity at different electrokinetic width with radius for different relaxation time $\bar{\lambda}_{1}$ when $\bar{t}=0.5$ and $a=1$. (a) $\bar{\lambda}_{1}=0.01$; (b) $\bar{\lambda}_{1}=0.1 ;$ (c) $\quad \bar{\lambda}_{1}=0.3 ;$ (d) $\bar{\lambda}_{1}=0.7$. 
relaxation time $\bar{\lambda}_{1}$, it is clearly seen from Figure 4(a) that as the electrokinetic width $K$ value increases, the normalized pulse EOF velocity variations are restricted to a very narrow area close to the EDL. However, with the increase of relaxation time $\bar{\lambda}_{1}$, the elasticity of the fluid becomes significant. And due to the elasticity of the physical property of the fluid as a whole, so the velocity variation can extend to the whole flow field (see Figures 4(b)-(d)) [46]. In addition, the amplitude of velocity increases with the gradual increase of relaxation time $\bar{\lambda}_{1}$.

\section{Conclusions}

A semi-analytical solution of the transient pulse EOF of Maxwell fluid through a circular micro-channel under the Debye-Hückel approximation is presented in this work. The computational results show that the velocity profiles depend mainly on the relaxation time $\bar{\lambda}_{1}$, the pulse width $a$ and the electrokinetic width $K$. With the aid of inverse Laplace transform, the following conclusions can be drawn:

- The profiles of normalized pulse EOF velocity vary rapidly and gradually stabilize as the increase of time $\bar{t}$ within a half period (that is, pulse width a).

- Increasing relaxation time $\bar{\lambda}_{1}$ will lead to larger velocity amplitude. In addition, the time needed to attain the steady status becomes longer with the increase of relaxation time $\bar{\lambda}_{1}$.

- The velocity profiles at the center of the micro-channel increase significantly with relaxation time $\bar{\lambda}_{1}$, especially for the smaller pulse width $a$. However, as the increase of pulse width $a$, this change will be less obvious. At the same time, the different change frequency of velocity profiles becomes slower, which means a long cycle time, and the time required for the fluid to reach a steady state becomes longer.

- With the increase of electrokinetic width $K$, the velocity variations are confined to a very narrow area close to the EDL for small relaxation time $\bar{\lambda}_{1}$. However, as the relaxation time $\bar{\lambda}_{1}$ increases, the elasticity of the fluid becomes conspicuous and the velocity variations can be extended to the entire region of flow.

\section{Acknowledgements}

This work was supported by the Scientific Research Project of Inner Mongolia University of Technology (Grant No. ZZ201813).

\section{Conflicts of Interest}

This manuscript has not been published and is not under consideration for publication elsewhere. We have no conflicts of interest to disclose.

\section{References}

[1] Stone, H.A., Stroock, A.D. and Ajdari, A. (2004) Engineering Flows in Small Devic- 
es: Microfluidics toward a Lab-on-a-Chip. Annual Review of Fluid Mechanics, 36, 381. https://doi.org/10.1146/annurev.fluid.36.050802.122124

[2] Bayraktar, T. and Pidugu, S.B. (2006) Characterization of Liquid Flows in Microfluidic Systems. International Journal of Heat and Mass Transfer, 49, 815. https://doi.org/10.1016/j.ijheatmasstransfer.2005.11.007

[3] Bao, L.P., Jian, Y.J., Chang, L., Su, J., Zhang, H.Y. and Liu, Q.S. (2013) Time Periodic Electroosmotic Flow of the Generalized Maxwell Fluids in a Semicircular Microchannel. Communications in Theoretical Physics, 59, 615-622. https://doi.org/10.1088/0253-6102/59/5/16

[4] Hunter, R.J. (1981) Zeta Potential in Colloid Science. Academic Press, Cambridge.

[5] Levine, S., Marriott, J.R., Neale, G. and Epstein, N. (1975) Theory of Electrokinetic Flow in Fine Cylindrical Capillaries at High Zeta Potentials. Journal of Colloid \& Interface Science, 52, 136-149. https://doi.org/10.1016/0021-9797(75)90310-0

[6] Taos, H.K. (2000) Electroosmotic Flow through an Annulus. Journal of Colloid \& Interface Science, 225, 247-250. https://doi.org/10.1006/jcis.1999.6696

[7] Hsu, J.P., Kao, C.Y., Tseng, S.J. and Chen, C.J. (2002) Electrokinetic Flow through an Elliptical Microchannel: Effects of Aspect Ratio and Electrical Boundary Conditions. Journal of Colloid \& Interface Science, 248, 176-184.

https://doi.org/10.1006/jcis.2001.8200

[8] Yang, C., Li, D. and Masliyah, J.H. (1998) Modeling Forced Liquid Convection in Rectangular Microchannels with Electrokinetic Effects. International Journal of Heat \& Mass Transfer, 41, 4229-4249. https://doi.org/10.1016/S0017-9310(98)00125-2

[9] Bianchi, F., Ferrigno, R. and Girault, H.H. (2000) Finite Element Simulation of an Electroosmotic Driven Flow Division at a t-Junction of Microscale Dimensions. Analytical Chemistry, 72, 1987-1993. https://doi.org/10.1021/ac991225z

[10] Wang, C.Y., Liu, Y.H. and Chang, C.C. (2008) Analytical Solution of Electro-Osmotic Flow in a Semicircular Microchannel. Physics of Fluids, 20, Article ID: 063105. https://doi.org/10.1063/1.2939399

[11] Dutta, P. and Beskok, A. (2001) Analytical Solution of Time Periodic Electroosmotic Flows: Analogies to Stokes' Second Problem. Analytical Chemistry, 73, 5097-5102. https://doi.org/10.1021/ac015546y

[12] Keh, H.J. and Tseng, H.C. (2001) Transient Electrokinetic Flow in Fine Capillaries. Journal of Colloid \& Interface Science, 242, 450-459. https://doi.org/10.1006/jcis.2001.7797

[13] Kang, Y.J., Yang, C. and Huang, X.Y. (2002) Dynamic Aspects of Electroosmotic Flow in a Cylindrical Microcapillary. International Journal of Engineering Science, 40, 2203-2221. https://doi.org/10.1016/S0020-7225(02)00143-X

[14] Wang, X.M., Chen, B. and Wu, J.K. (2007) A Semianalytical Solution of Periodical Electro-Osmosis in a Rectangular Microchannel. Physics of Fluids, 19, Article ID: 127101. https://doi.org/10.1063/1.2784532

[15] Chakraborty, S. and Ray, S. (2008) Mass Flow-Rate Control through Time Periodic Electro-Osmotic Flows in Circular Microchannels. Physics of Fluids, 20, Article ID: 083602. https://doi.org/10.1063/1.2949306

[16] Jian, Y.J., Yang, L.G. and Liu, Q.S. (2010) Time Periodic Electro-Osmotic Flow through a Microannulus. Physics of Fluids, 22, Article ID: 042001. https://doi.org/10.1063/1.3358473

[17] Deng, S.Y., jian, Y.J., Bi, Y.H., Chang, L., Wang, H.J. and Liu, Q.S. (2010) Unsteady 
Electroosmotic Flow of Power-Law Fluid in a Rectangular Microchannel. Mechanics Research Communications, 39, 9-14. https://doi.org/10.1016/j.mechrescom.2011.09.003

[18] Zhao, M.L. and Wang, S.W. (2015) Analytical Solution of the Transient Electro-Osmotic Flow of a Generalized Fractional Maxwell Fluid in a Straight Pipe with a Circular Cross-Section. European Journal of Mechanics-B/ Fluids, 54, 82-86. https://doi.org/10.1016/j.euromechflu.2015.06.016

[19] Jian, Y.J., Su, J., Chang, L., Liu, Q.S. and He, G.W. (2014) Transient Electroosmotic Flow of General Maxwell Fluids through a Slit Microchannel. Zeitschrift fur Angewandte Mathematik und Physik (ZAMP), 65, 435-447. https://doi.org/10.1007/s00033-013-0341-1

[20] Zhao, M.L., Wang, S.W. and Wei, S.S. (2013) Transient Electro-Osmotic Flow of Oldroyd-B Fluid in a Straight Pipe of Circular Cross Section. Journal of Non-Newtonian Fluid Mechanics, 201, 135-139. https://doi.org/10.1016/j.jnnfm.2013.09.002

[21] Jiang, Y.T., Qi, H.T., Xu, H.Y. and Jiang, X.Y. (2017) Transient Electroosmotic Slip Flow of Fractional Oldroyd-B Fluids. Microfluidics and Nanofluidics, 21, 1-10. https://doi.org/10.1007/s10404-016-1843-x

[22] Liang, P.C., Wang, S.W. and Zhao, M.L. (2020) Numerical Study of Rotating Electroosmotic Flow of Oldroyd-B Fluid in a Microchannel with Slip Boundary Condition. Chinese Journal of Physics, 65, 459-471. https://doi.org/10.1016/j.cjph.2020.02.025

[23] Xie, Z.Y. and Jian, Y.J. (2014) Rotating Electroosmotic Flow of Power-Law Fluids at High Zeta Potentials. Colloids \& Surfaces A Physicochemical \& Engineering Aspects, 461, 231-239. https://doi.org/10.1016/j.colsurfa.2014.07.051

[24] Baos, R., Arcos, J., Bautista, O. and Mendez, F. (2018) Oscillatory Electroosmotic Flow of Power-Law Fluids in a Microchannel. International Journal of Mechanical \& Mechatronics, 12, 773-776.

[25] Baos, R., Arcos, J., Bautista, O. and Mendez, F. (2020) Slippage Effect on the Oscillatory Electroosmotic Flow of Power-Law Fluids in a Microchannel. Defect and Diffusion Forum, 399, 92-101. https://doi.org/10.4028/www.scientific.net/DDF.399.92

[26] Sun, L.X., Jian, Y.J., Chang, L., Zhang, H.Y. and Liu, Q.S. (2013) Alternating Current Electro-Osmotic Flow of the Maxwell Fluids through a Circular Micro-Pipe. Journal of Mechanics, 29, 233-240. https://doi.org/10.1017/jmech.2012.138

[27] Adegbie, K.S., Omowaye, A.J., Disu, A.B. and Animasaun, I.L. (2015) Heat and Mass Transfer of Upper Convected Maxwell Fluid Flow with Variable Thermo-Physical Properties over a Horizontal Melting Surface. Applied Mathematics, 6, 1362-1379. https://doi.org/10.4236/am.2015.68129

[28] Animasaun, I.L. and Omowaye, A.J. (2016) Upper-Convected Maxwell Fluid Flow with Variable Thermo-Physical Properties over a Melting Surface Situated in Hot Environment Subject to Thermal Stratification. Journal of Applied Fluid Mechanics, 9, 1777-1790. https://doi.org/10.18869/acadpub.jafm.68.235.24939

[29] Koríko, O.K., Adegbie, K.S., Omowaye, A.J. and Animasaun, I.L. (2016) Boundary Layer Analysis of Upper Convected Maxwell Fluid Flow with Variable Thermo-Physical Properties over a Melting Thermally Stratified Surface. FUTA Journal of Research in Sciences, 12, 287-298.

[30] Chu, X. and Jian, Y.J. (2019) Magnetohydrodynamic Electroosmotic Flow of Maxwell Fluids with Patterned Charged Surface in Narrow Confinements. Journal of Physics D Applied Physics, 52, Article ID: 405003. 
https://doi.org/10.1088/1361-6463/ab2b27

[31] Escandón, J., Torres, D., Hernández, C. and Vargas, R. (2020) Start-Up Electroosmotic Flow of Multi-Layer Immiscible Maxwell Fluids in a Slit Microchannel. Micromachines, 11, 757. https://doi.org/10.3390/mi11080757

[32] Shah, N.A., Mahsud, Y., Aziz, M. and Tlili, I. (2020) Analytical Solutions for Unsteady Electrohydrodynamics Flows of Maxwell Fluids in Microchannels with Circular Cross Section. Physics of Fluids, 32, Article ID: 013107. https://doi.org/10.1063/1.5128688

[33] Liu, Q.S., Jian, Y.J. and Yang, L.G. (2011) Alternating Current Electroosmotic Flow of the Jeffreys Fluids through a Slit Microchannel. Physics of Fluids, 23, 381. https://doi.org/10.1063/1.3640082

[34] Gao, C.H. and Jian, Y.J. (2015) Analytical Solution of Magnetohydrodynamic Flow of Jeffrey Fluid through a Circular Microchannel. Journal of Molecular Liquids, 211, 803-811. https://doi.org/10.1016/j.molliq.2015.08.004

[35] Li, F.Q., Jian, Y.J., Xie, Z.Y., Liu, Y.B. and Liu, Q.S. (2017) Transient Alternating Current Electroosmotic Flow of a Jeffrey Fluid through a Polyelectrolyte-Grafted Nanochannel. RSC Advances, 7, 782-790. https://doi.org/10.1039/C6RA24930B

[36] Fan, Y. and Cheng, Q. (2013) Analysis of Current on Human Therapeutic Effects in Low Frequency Pulse. Journal of Henan Institute of Science and Technology, 41, 45-47.

[37] Liu, Q.S., Jian, Y.J., Chang, L. and Yang, L.G. (2012) Alternating Current (AC) Electroosmotic Flow of Generalized Maxwell Fluids through a Circular Microtube. International Journal of Physical Sciences, 7, 5935-5941.

[38] Zhang, X., Zhang, X., Liu, Z., Tao, C. and Quan, X. (2019) Pulse Current Electrodeposition of Manganese Metal from Sulfate Solution. Journal of Environmental Chemical Engineering, 7, Article ID: 103010. https://doi.org/10.1016/j.jece.2019.103010

[39] Li, C., Yun, T., Xu, J., Li, F. and Bao, H. (2020) Effect of Pulse Current on Bending Springback of Nanocrystalline Ni Foil. Journal of Materials Engineering and Performance, 29, 2368-2373. https://doi.org/10.1007/s11665-020-04761-6

[40] Pujiyulianto, E. and Suyitno (2021) Effect of Pulse Current in Manufacturing of Cardiovascular Stent Using EDM Die-Sinking. The International Journal of Advanced Manufacturing Technology, 112, 1-9. https://doi.org/10.1007/s00170-020-06484-3

[41] Yin, Z. (2014) Unsteady Electroosmotic Flow of Maxwell Fluids through a Parallel Plate Micro-Channel. Inner Mongolia University, Hohhot.

[42] Bird, R.B., Stewart, W.E. and Lightfoot, E.N. (2002) Transport Phenomena, Second Edition. John Wiley \& Sons, Inc., New York.

[43] Na, R., Jian, Y.J., Chang, L., Su, J. and Liu, Q.S. (2013) Transient Electro-Osmotic and Pressure Driven Flows through a Microannulus. Open Journal of Fluid Dynamics, 3, 50-56. https://doi.org/10.4236/ojfd.2013.32007

[44] Hoog, F.D., Knight, J.H. and Stokes, A.N. (1982) An Improved Method for Numerical Inversion of Laplace Transforms. SIAM Journal on Scientific and Statistical, 3, 357-366. https://doi.org/10.1137/0903022

[45] Li, X.X., Yin, Z., Jian, Y.J., Chang, L., Su, J. and Liu, Q.S. (2012) Transient Electro-Osmotic Flow of Generalized Maxwell Fluids through a Microchannel. Journal of Non-Newtonian Fluid Mechanics, 187-188, 43-47.

https://doi.org/10.1016/j.jnnfm.2012.09.005 
[46] Liu, Q.S., Jian, Y.J. and Yang, L.G. (2011) Time Periodic Electroosmotic Flow of the Generalized Maxwell Fluids between Two Micro-Parallel Plates. Journal of Non-Newtonian Fluid Mechanics, 166, 478-486.

https://doi.org/10.1016/j.jnnfm.2011.02.003

\section{Appendix}

The Laplace transform of $f(\bar{t})$ is expressed as follows

$$
\begin{aligned}
\int_{0}^{\infty} f(\bar{t}) \mathrm{e}^{-s \bar{t}} \mathrm{~d} \bar{t} & =\int_{0}^{T} f(\bar{t}) \mathrm{e}^{-s \bar{t}} \mathrm{~d} \bar{t}+\int_{T}^{\infty} f(\bar{t}) \mathrm{e}^{-s \bar{t}} \mathrm{~d} \bar{t} \\
& =\int_{0}^{T} f(\bar{t}) \mathrm{e}^{-s \bar{t}} \mathrm{~d} \bar{t}+\mathrm{e}^{-s T} \int_{0}^{\infty} f(\tau) \mathrm{e}^{-s \tau} \mathrm{d} \tau \quad(\text { Let } \bar{t}=T+\tau)
\end{aligned}
$$

here $T=2 a$ denotes the period of the ideal rectangle pulse wave.

By shifting the term of Equation (A.1) and using the Equation (1), we have

$$
\begin{aligned}
F(s) & =\int_{0}^{\infty} f(\bar{t}) \mathrm{e}^{-s \bar{t}} \mathrm{~d} \bar{t}=\frac{1}{1-\mathrm{e}^{-s T}} \int_{0}^{T} f(\bar{t}) \mathrm{e}^{-s \bar{t}} \mathrm{~d} \bar{t} \\
& =\frac{1}{1-\mathrm{e}^{-2 a s}} F_{1}(s)=\frac{1}{s} \tanh \left(\frac{a s}{2}\right)
\end{aligned}
$$

where

$$
\begin{aligned}
F_{1}(s) & =\int_{0}^{a} f(\bar{t}) \mathrm{e}^{-s \bar{t}} \mathrm{~d} \bar{t}+\int_{a}^{2 a} f(\bar{t}) \mathrm{e}^{-s \bar{t}} \mathrm{~d} \bar{t} \\
& =\frac{1-\mathrm{e}^{-a s}}{s}-\frac{\mathrm{e}^{-2 a s}\left(\mathrm{e}^{a s}-1\right)}{s} \\
& =\frac{\mathrm{e}^{-2 a s}\left(\mathrm{e}^{a s}-1\right)^{2}}{s}
\end{aligned}
$$

